\title{
Axial Localization Improvements when Trapping Aerosol Droplets using an Annular Beam
}

\author{
R D Dear ${ }^{1}$, D R Burnham², M D Summers ${ }^{1}$, D McGloin ${ }^{3}$, G A D Ritchie ${ }^{1}$ \\ 1.University of Oxford, Department of Chemistry, PTCL, South Parks Road, Oxford, OX1 3QZ \\ 2.Delft University of Technology, Department of Bionanoscience, Kavli Institute, Delft, The Netherlands \\ 3.University of Dundee, Division of Electronic Engineering and Physics, Nethergate, Dundee, DD1 4HN \\ richard.dear@chem.ox.ac.uk
}

\begin{abstract}
We demonstrate that annular beam traps lead to an increased size range and improved axial trapping of aerosols in comparison to Gaussian beam traps. These methods are extended to consider ionic liquid samples.

OCIS codes: (350.4855) Optical tweezers or optical manipulation; (010.1110) Aerosols
\end{abstract}

\section{Optical trapping using an annular beam}

In this work we quantify the improvements that can be achieved by using an annular beam trap, formed by blocking the centre of a Gaussian beam [1]. These improvements include an increase in the range of trappable particles, both in terms of the refractive index contrast between the trapping medium and particle, with particular applications in aerosol trapping where this contrast is large, and also an increase in the size range of trappable salt water aerosol droplets. In addition annular beams show improved axial localization and an increase in axial:lateral trap stiffness ratio. These improvements show excellent agreement with theoretical models of these systems [2], using a modification of Mie-Debye spherical aberration theory [3,4].

\section{Micro-viscosity measurements in ionic liquids}

Using a combination of high-speed camera tracking and back focal plane interferometry we have extended this work to include an investigation into the trapping of both ionic liquid droplets, defined as organic salts with a melting point below $100{ }^{\circ} \mathrm{C}$, and of tracer particles in ionic liquid samples. These methods have allowed us to monitor micro-viscosity changes under a variety of different conditions in these novel media.

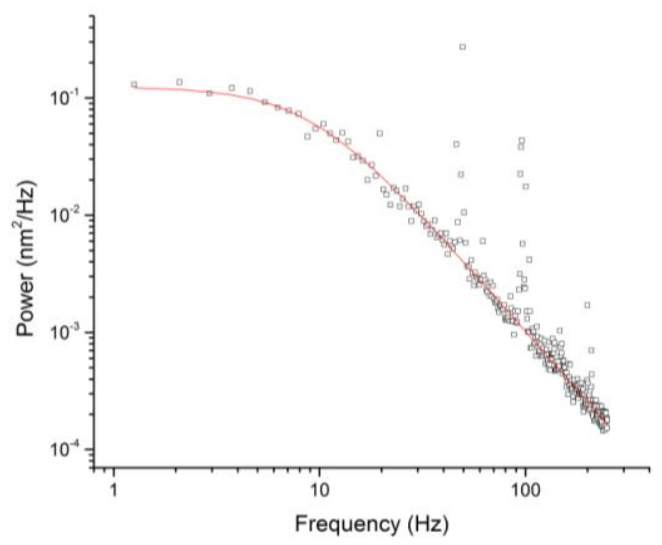

Fig 1. An example power spectrum for a $2.35 \mu \mathrm{m}$ melamine tracer particle trapped in ethylammonium nitrate.

\section{References}

[1] Dear RD, Burnham DR, Summers MD, McGloin D, Ritchie G A D, "Single aerosol trapping with an annular beam: improved particle localization" Phys. Chem. Chem. Phys., 14, 15826-31 (2012).

[2] Burnham DR, McGloin D, "Modeling of optical traps for aerosols” Journal of the Optical Society of America B, 28(12), 2856-64 (2011).

[3] Mazolli A, Maia Neto PA, Nussenzveig HM, "Theory of trapping forces in optical tweezers" Proceedings of the Royal Society A: Mathematical, Physical and Engineering Sciences, 459(2040), 3021-41 (2003).

[4] Viana NB, Rocha MS, Mesquita ON, Mazolli A., Maia Neto P A., Nussenzveig HM, "Towards absolute calibration of optical tweezers" Physical Review E, 75(2), 1-14 (2007). 